\title{
Stacking of InAs/InP(001) quantum wires studied by in situ stress measurements: Role of inhomogeneous stress fields
}

\author{
David Fuster, ${ }^{a)}$ María Ujué González, Luisa González, and Yolanda González \\ Instituto de Microelectrónica de Madrid (CNM-CSIC), Tres Cantos, Madrid, Spain \\ Teresa Ben, Arturo Ponce, and Sergio I. Molina \\ Departamento de Ciencia de los Materiales e I. M. y Q. I., Universidad de Cádiz, Puerto Real, \\ Cádiz, Spain
}

(Received 2 February 2004; accepted 7 April 2004; published online 20 May 2004)

\begin{abstract}
Size and spatial distribution homogeneity of nanostructures is greatly improved by making stacks of nanostructures separated by thin spacers. In this work, we present in situ and in real time stress measurements and reflection high-energy electron diffraction observations and ex situ transmission electron microscopy (TEM) characterization of stacked layers of InAs quantum wires (QWRs) separated by InP spacer layers, $d(\mathrm{InP})$, of thickness between 3 and $20 \mathrm{~nm}$. For $d(\mathrm{InP})<20 \mathrm{~nm}$, the amount of InAs involved in the created QWR from the second stack layer on, exceeds that provided by the In cell. Our results suggest that in those cases InAs three dimensional islands formation starts at the P/As switching and lasts during further InAs deposition. We propose an explanation for this process that is strongly supported on TEM observations. The results obtained in this work imply that concepts like the existence of a critical thickness for two- to three-dimensional growth mode transition should be revised in correlated QWR stacks of layers. (C) 2004 American Institute of Physics. [DOI: 10.1063/1.1759374]
\end{abstract}

The incorporation of nanostructures in electronic and optoelectronic devices provides properties improvement and design possibilities, yet requiring the control of their size and position. For self-assembled nanostructures, the size homogeneity and spatial distribution can be greatly improved by stacking several layers. ${ }^{1-4}$ In this context, the vertical stacks of self-assembled InAs quantum wires (QWRs) grown on $\mathrm{InP}(001)$ are of particular interest for lasers, as they emit light at 1.30 and $1.55 \mu \mathrm{m} .^{5-7}$

In stacks of nanostructures, the buried ones produce inhomogeneous strain fields that propagate toward the capping layer surface where the next nanostructures will be formed. ${ }^{1,2}$ This leads to a vertical correlation between the nanostructures layers depending both on the size of the buried nanostructures and the spacer layer thickness.

In this work, we have studied the growth of multilayers of InAs QWRs with different spacer layer thicknesses, $d(\mathrm{InP})$, by in situ and in real time stress measurements and reflection high-energy electron diffraction (RHEED). A strong influence of the spacer layer thickness on QWR formation process in the second and successive layers of the stack has been observed: QWRs are correlated for $d(\mathrm{InP})<20 \mathrm{~nm}$, where a reduction of the amount of deposited InAs necessary for QWR formation (observed by RHEED) together with an increase in InAs growth rate (obtained from stress measurements) take place. In order to explain these results, we propose a model that is strongly supported by transmission electron microscopy (TEM) images. Our experiments provide quantitative values of the extra amount of material involved in the formation of vertically correlated self-assembled nanostructures, as well as the evi-

${ }^{\text {a)} E l e c t r o n i c ~ m a i l: ~ d a v i d f @ i m m . c n m . c s i c . e s ~}$ dence of the absence of a two-dimensional (2D)-threedimensional (3D) growth mode transition as the relevant process in nanostructures self-assembling during stacking.

The samples under study consist of stacks of two layers of InAs QWRs grown by solid-source molecular-beam epitaxy (MBE) separated by InP spacer layers of different thickness $[d(\mathrm{InP})=3,5,10$, and $20 \mathrm{~nm}]$. The QWRs were formed by deposition of 2.5 monolayers (ML) of InAs at $0.1 \mathrm{ML} / \mathrm{s}$, growth temperature $T_{s}=515^{\circ} \mathrm{C}$ and beam equivalent pressure $\left(\mathrm{As}_{4}\right)=2.3 \times 10^{-6}$ mbar. The InP spacer layer was grown at $380{ }^{\circ} \mathrm{C}$ by atomic layer $\mathrm{MBE}$ at $1 \mathrm{ML} / \mathrm{s}$. The resulting QWRs are oriented along $[1 \overline{10}]$ and periodically arranged along the [110] direction. ${ }^{5}$

QWR formation, and thus InAs critical thickness, $\theta_{c}$, has been detected in situ by the emergence of a characteristic 3D RHEED pattern. Stress evolution during this process has been obtained by optical monitorization of the substrate curvature. $^{8-10}$ This technique provides a direct, in situ, and in real time measurement of the film accumulated stress, $\Sigma \sigma$ (stress integrated along the layer thickness). ${ }^{8,10}$ We use thinned $\operatorname{InP}(001)$ substrates $(190 \mu \mathrm{m})$ to improve sensitivity, elongated along $[1 \overline{1} 0]$ to detect stress variations in this direction. Cross-sectional TEM experiments were carried out in a JEOL 1200EX $(120 \mathrm{kV})$.

The observation of the 2D-3D transition by RHEED reveals that, depending on InP spacer thickness, the InAs critical thickness for QWR formation, $\theta_{c}$, drastically decreases at the second layer of the QWR stack, as was previously reported for InAs/GaAs. ${ }^{11}$ In Fig. 1 we show, in full squares, the difference in $\theta_{c}\left(\Delta \theta_{c}\right)$ between the first and second layer of the QWR stack as a function of the thickness of the InP spacer that separates both QWR layers. (The error bars in the graph account for the inaccuracy in the visual 


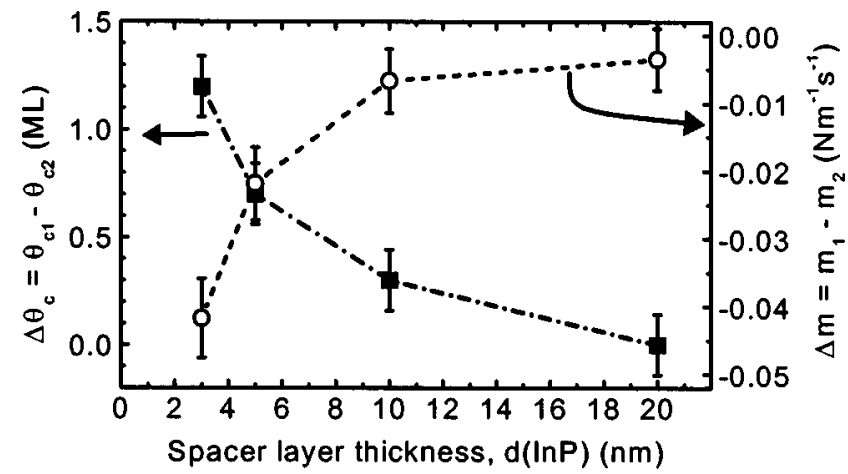

FIG. 1. Difference in InAs critical thickness for QWR formation between the first and second layer, $\Delta \theta_{c}=\theta_{c 1}-\theta_{c 2}$ (full squares), and in slope of accumulated stress $\Delta m=m_{1}-m_{2}$ (empty dots), for stacked layers with different InP spacer thickness.

determination of the 3D RHEED pattern emergence). For $d(\mathrm{InP})=20 \mathrm{~nm}$, no clear change is observed for $\Delta \theta_{c}$ within the experimental error. This means that, in the InAs/InP system, a spacer layer thickness $d(\mathrm{InP})=20 \mathrm{~nm}$ is thick enough to cancel the influence of the stress field of the buried QWR.

Figure 2 shows the evolution of the accumulated stress, $\Sigma \sigma$, in [1 10$]$ direction during InAs deposition for bilayers of QWR with $d(\operatorname{InP})=3,5,10$, and $20 \mathrm{~nm}$. In all cases, we observe a linear increase in $\Sigma \sigma$, indicating pseudomorphic growth along $[1 \overline{1} 0] .{ }^{8}$ This behavior is in complete agreement with QWR formation, which as a result of their geometry relax stress only in the direction perpendicular to their orientation and, so far, no stress relaxation due to QWR formation can be measured in [1 $1 \overline{1} 0]$ direction. ${ }^{9,12}$ Accordingly, $\Sigma \sigma$ evolution in $[1 \overline{10}]$ corresponds unequivocally to the total amount of InAs grown.

From the data plotted in Fig. 2, it is evident that during InAs deposition $\Sigma \sigma$ slope due to InAs growth is larger in the

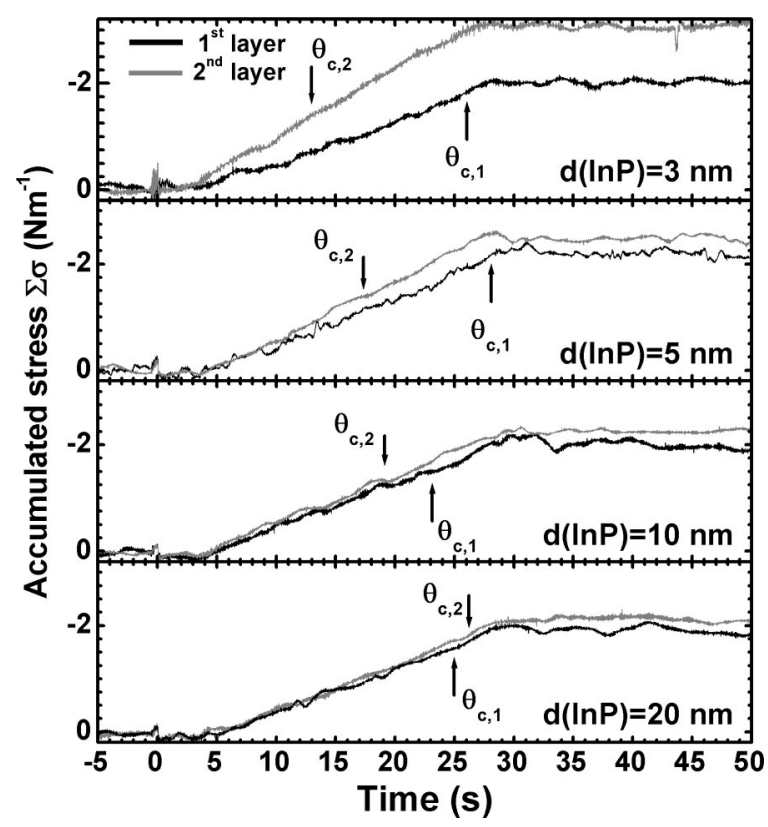

FIG. 2. Accumulated stress $\left(\sum \sigma\right)$ along $[1 \overline{1} 0]$ direction during InAs deposition at $0.1 \mathrm{ML} / \mathrm{s}$ for growth of two stacked QWR layers separated by different InP spacer layers thickness $[d(\mathrm{InP})=3,5,10$, and $20 \mathrm{~nm}]$. Critical thickness for QWR formation detected by RHEED, $\theta_{c}$, is marked with

relevant changes in $\sum \sigma$ are observed. This result was ex-
arrows in all cases.
Downloaded 04 May 2010 to 161.111.235.252. Redistribution subject to AIP license or copyright; see http://apl.aip.org/apl/copyright.jsp
TABLE I. Relevant parameters in QWR formation for stacked layers with different InP spacers, $d(\mathrm{InP})$ : Difference between the first and second layers in InAs critical thickness of the stack, $\Delta \theta_{c}=\theta_{c 1}-\theta_{c 2}$, and in slopes of the accumulated stress, $\Delta m=m_{1}-m_{2}$; and excess of InAs per deposited monolayer grown at the second layer, $E(\operatorname{InAs})$

\begin{tabular}{cccccc}
\hline \hline $\begin{array}{c}d(\mathrm{InP}) \\
(\mathrm{nm})\end{array}$ & $\begin{array}{c}\Delta \theta_{c} \\
(\mathrm{ML})\end{array}$ & $\begin{array}{c}m_{1} \\
(\mathrm{~N} / \mathrm{ms})\end{array}$ & $\begin{array}{c}m_{2} \\
(\mathrm{~N} / \mathrm{ms})\end{array}$ & $\begin{array}{c}\Delta m \\
(\mathrm{~N} / \mathrm{ms})\end{array}$ & $\begin{array}{c}E(\mathrm{InAs}) \\
(\mathrm{ML})\end{array}$ \\
\hline 3 & 1.2 & $0.084 \pm 0.003$ & $0.126 \pm 0.005$ & -0.042 & $0.50 \pm 0.08$ \\
5 & 0.7 & $0.088 \pm 0.003$ & $0.110 \pm 0.004$ & -0.022 & $0.26 \pm 0.07$ \\
10 & 0.3 & $0.083 \pm 0.003$ & $0.089 \pm 0.004$ & -0.006 & $0.08 \pm 0.06$ \\
20 & 0 & $0.081 \pm 0.003$ & $0.085 \pm 0.003$ & -0.004 & $0.04 \pm 0.06$ \\
\hline
\end{tabular}

second QWR layer than in the first one [it only coincides for $d(\operatorname{InP})=20 \mathrm{~nm}]$. Moreover, $\Sigma \sigma$ slope for the first layer is the same in all cases (see also Table I), as expected since it corresponds to identical experiments (InAs deposited on "plain" InP). However, during InAs deposition on InP with buried QWR (second layer), the amount of the built-in $\Sigma \sigma$ is higher for decreasing $d(\mathrm{InP})$. Given that for $d(\mathrm{InP})=20 \mathrm{~nm}$ $\Sigma \sigma$ slope is the same for the first and second layers, this proves that the stress fields due to the buried QWR decays significantly at this distance and has no influence on the formation of the subsequent QWR, in agreement with RHEED critical thickness data (Fig. 1 and Table I).

Using InAs and InP bulk elastic constants, the estimated value for the accumulated stress corresponding to $1 \mathrm{ML}$ of InAs on $\operatorname{InP}(001)$ is $\Sigma \sigma=0.78 \mathrm{Nm}^{-1}$, which gives a $\Sigma \sigma$ slope of $0.078 \mathrm{Nm}^{-1} \mathrm{~s}^{-1}$ for $0.1 \mathrm{ML} / \mathrm{s} \mathrm{InAs} \mathrm{growth} \mathrm{rate.}$ This is in good agreement with the experimental average value $m_{1}=0.084 \mathrm{Nm}^{-1} \mathrm{~s}^{-1}$ for InAs deposition in the first QWR layers (notice that bulk values may not be totally correct for only two atomic layers). However, for the second layers, $\Sigma \sigma$ slope $\left(\mathrm{m}_{2}\right)$ is higher. This means that, from the onset of InAs deposition, the actual InAs growth rate is higher than the InAs deposition rate (fixed by the In flux delivered by the In cell). Furthermore, as the thinner the spacer layer is, the larger $m_{2}$, the actual InAs growth rate increases as $d(\mathrm{InP})$ decreases. For example, we obtain a $m_{2} / m_{1}$ ratio of 1.5 for $d(\mathrm{InP})=3 \mathrm{~nm}$, which means that total InAs grown is 1.5 times the InAs deposited or, in other words, that the excess of InAs grown per deposited monolayer is 0.5 ML (see Table I). Consequently, in correlated nanostructures stacks, the amount of material deposited for nanostructures formation should be adjusted at each layer in order to improve size uniformity.

The main difference between a plain InP surface and a surface of InP with QWR below is that in the last case, the buried QWR produces an inhomogeneous strain field that propagates toward the surface. So, stress driven processes must play an important role in the formation of excess of InAs. ${ }^{13-17}$ Accordingly, we propose that InAs grows faster at the low strained areas of the surface, process that involves In tion, we have measured $\Sigma \sigma$ evolution in similar experiments but exposing the InP surface only to $\mathrm{As}_{4}$ flux at $515^{\circ} \mathrm{C}$.

Figure 3 shows the results obtained for $\Sigma \sigma$ along [1 $\overline{1} 0]$ with $\mathrm{As}_{4}$ flux impinging on a InP plain surface (solid line), and InP surfaces with buried QWR at a distance of 5 and 3 $\mathrm{nm}$. Without a surface strain field due to buried QWR, no relevant changes in $\Sigma \sigma$ are observed. This result was exmigration and As/P exchange. In order to prove this assump- 


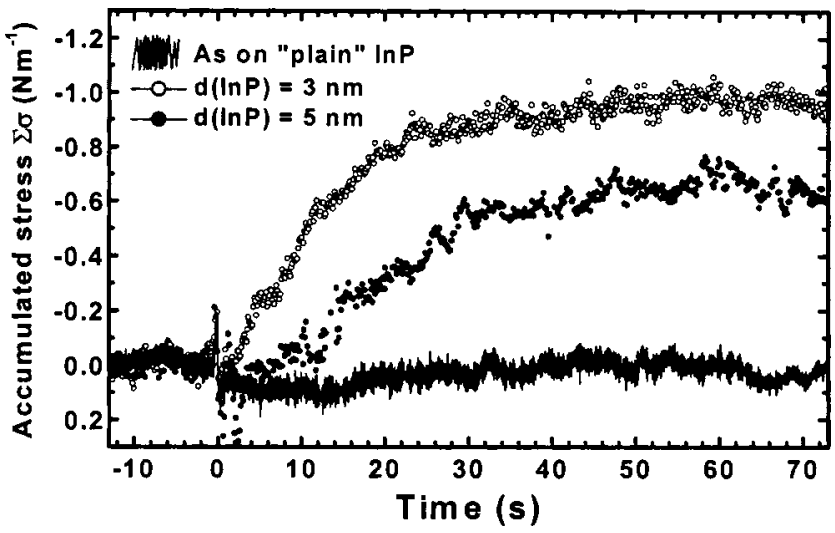

FIG. 3. Accumulated stress evolution measured when exposing to arsenic flux a plain InP (solid line) and InP surfaces separated from buried QWR by 5 (full dots) and $3 \mathrm{~nm}$ (empty dots).

pected because As/P exchange process on $\operatorname{InP}(001)$ saturates very fast $(\tau<1 \mathrm{~s})$ when the reaction occurs homogeneously at the surface, forming one 2D-InAs strained layer that does not incorporate stress along [1 $\overline{1} 0]$ (solid line on Fig. 3). ${ }^{17}$

However, when the buried QWR exist close to the surface, an increase of compressive $\Sigma \sigma$ is measured that corresponds to the growth of InAs on top of that InAs 2D layer produced by As/P exchange, even when no In has been deposited (dots in Fig. 3). Moreover, few seconds after the P/As switching a 3D RHEED pattern appears that corresponds to the QWR formation.

These results suggest that immediately after P/As switching, 3D-InAs starts growing at the low strained InAs areas of the surface. This implies mass transport from the 2D-InAs formed by As/P exchange to form the 3D islands that will evolve to QWR. This process leaves in the vicinity of the InAs 3D islands bare InP depleted regions exposed to an $\mathrm{As}_{4}$ flux. A quick $\mathrm{P}$ replacement by As leads again to 2D InAs that act as continuous source for further QWR formation and enlargement (see Fig. 4).

The above proposed process should produce a typical morphology consisting of dips between QWRs formed on top of plateaus. This is just what we obtain by cross-sectional TEM (Fig. 5) from a four layer QWR stack with $d(\mathrm{InP})=5$

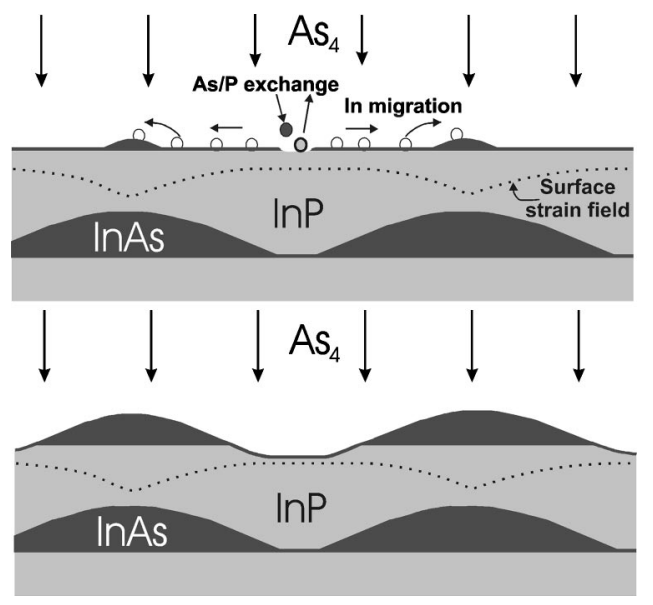

FIG. 4. Proposed process for InAs growth from InP exposed at $\mathrm{As}_{4}$ flux (without In deposition) due to the strain induced by the buried QWRs.

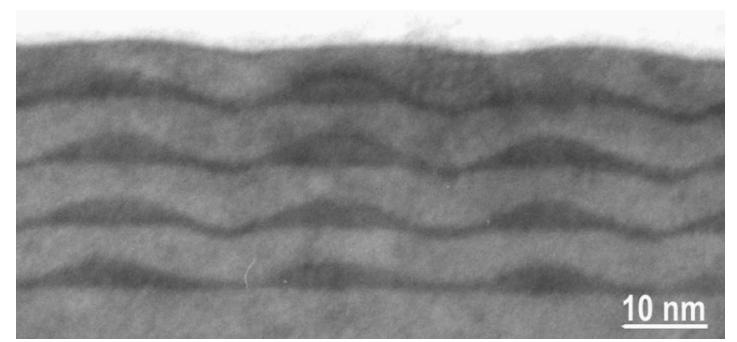

FIG. 5. Cross-sectional TEM image of a sample consisting of four stacked QWRs, showing the InAs (dark) and InP (bright) material.

nm grown under similar conditions. We observe in the second and next layers the QWR mounted on flat areas separated by undulated valleys, while a flat interface is seen in the first QWR layer. In this context, the "critical thickness" determined by the onset of a 3D RHEED pattern just reflects that the $3 \mathrm{D}$ islands have reached a certain size detectable by this technique.

In summary, we have measured the enhancement of InAs growth driven by stress processes. Our in situ results imply that in the presence of inhomogeneous strain, 3D islands are just formed at the P/As switching, even without In deposition. This indicates that other process rather than a 2D-3D growth mode-transition at a certain critical thickness are needed to describe this system. Instead, we explain the formation of the QWR in correlated stack layers considering stress driven mass transport together with an efficient P/As exchange. Our model is strongly supported by TEM.

This work was financed by Spanish MCyT under NANOSELF project (TIC2002-04096), by NANOMAT project of the EC Growth Program (No. G5RD-CT-200100545) by Junta de Andalucía (TEP-0120). TEM measurements were carried out in the DME-SCCYT and UCM.

${ }^{1}$ Q. Xie, A. Madhukar, P. Chen, and N. P. Kobayashi, Phys. Rev. Lett. 75, 2542 (1995)

${ }^{2}$ J. Tersoff, C. Teichert, and M. G. Lagally, Phys. Rev. Lett. 76, 1675 (1996).

${ }^{3}$ B. Lita, R. S. Goldman, J. D. Philips, and P. K. Bhattacharya, Appl. Phys. Lett. 74, 2824 (1999).

${ }^{4}$ B. Alén, J. Martinez-Pastor, L. González, J. M. García, S. I. Molina, A. Ponce, and R. García, Phys. Rev. B 65, 241301 (2002).

${ }^{5}$ L. González, J. M. García, R. García, F. Briones, J. Martínez-Pastor, and C. Ballesteros, Appl. Phys. Lett. 76, 1104 (2000).

${ }^{6}$ B. Alén, J. Martínez-Pastor, A. García-Cristóbal, L. González, and J. M. García, Appl. Phys. Lett. 78, 4025 (2001).

${ }^{7}$ X. Mu, I. B. Zotova, Y. J. Ding, H. Yang, and G. J. Salamo, Appl. Phys. Lett. 79, 1091 (2001).

${ }^{8}$ M. U. González, Y. González, and L. González, Appl. Phys. Lett. 81, 4162 (2002).

${ }^{9}$ M. U. González, L. González, J. M. García, Y. González, J. P. Silveira, and F. Briones, Microelectron. J. 35, 13 (2004).

${ }^{10}$ C. A. Volkert, J. Appl. Phys. 70, 3521 (1991).

${ }^{11}$ Y. Nakata, Y. Sugiyama, T. Futatsugi, and N. Yokoyama, J. Cryst. Growth 175, 713 (1997).

${ }^{12}$ J. M. García, L. González, M. U. González, J. P. Silveira, Y. González, and F. Briones, J. Cryst. Growth 227, 975 (2001).

${ }^{13}$ H. R. Gutiérrez, M. A. Cotta, J. R. R. Bortoleto, and M. M. G. De Carvalho, J. Appl. Phys. 92, 7523 (2002).

14 Wang, F. Zhao, Y. Peng, Z. Jin, Y. Li, and S. Liu, Appl. Phys. Lett. 72, 2433 (1998).

${ }^{15}$ S. Yoon, Y. Moon, T. Lee, and E. Yoon, Appl. Phys. Lett. 74, 2029 (1999).

${ }^{16}$ H. Yang, P. Ballet, and G. J. Salamo, J. Appl. Phys. 89, 7871 (2001).

${ }^{17}$ M. U. González, J. M. García, L. González, J. P. Silveira, Y. González, J. D. Gómez, and F. Briones, Appl. Surf. Sci. 188, 188 (2002). 